\title{
Analysis of Rhythmic Rectal Contractions during Filling Cystometry in Women
}

\author{
Françoise A. Valentini, Brigitte G. Marti, Gilberte Robain, Pierre P. Nelson
}

UMRS 731 (FAV, GR), INSERM / Université Pierre et Marie Curie (Université Paris 6), Paris, France and Service de Médecine Physique et Réadaptation (FAV, BGM, GR, PPN), Hopital Charles Foix, Ivry-sur-Seine, France

\begin{abstract}
Purpose: Rhythmic or random rectal contractions independent of bladder activity are frequently observed during cystometry and usually attributed either to a neurological disease, or to ageing. The aim of our study was to search for an association of rhythmic rectal contractions (RRCs) with a specific lower urinary tract symptom or/and an urodynamic diagnosis.

Materials and Methods: The population consisted of 534 consecutive women with lower urinary tract symptoms and without specific gastro-intestinal disease referred for urodynamics; 382 (non-ND) had no history of neurological disease and 152 (ND) a history of neurological disease. Cystometries were performed according to ICS recommendations. Rectal pressure was measured using a punctured balloon filled with $2 \mathrm{~mL}$ of saline. RRCs were defined as rhythmic changes in the rectal pressure of at least $3 \mathrm{~cm} \mathrm{H} 2 \mathrm{O}$ independent of the total vesical pressure.

Results: RRCs were observed in 69 patients, with no difference in neurological status or age (non-ND: 12.3\% and 65.5y; ND: $14.5 \%$ and $62.7 y)$. Patients with RRCs were significantly older than the negative population $(p=0.0002)$. RRCs had a low frequency: $1-4 / \mathrm{min}$; their amplitude was $\leq 15 \mathrm{~cm} \mathrm{H} 2 \mathrm{O}$ in 67 patients. RRCs were associated with urgency $(35$ patients) whatever the neurological status and with detrusor overactivity only in the neurological patients.

Conclusions: RRCs cannot be considered as artefactual events during cystometry in women, occur in the older population, are frequently associated with urgency but not with detrusor overactivity or neurological disease. Occurrence of RRCs should prompt the physician to look at the possible causes of urgency (colonic or bladder).
\end{abstract}

Key words: urodynamics; rectum; urinary tract diseases; women

Int Braz.J Urol. 2009; 35: 475-83

\section{INTRODUCTION}

Rhythmic or random rectal contractions, independent of bladder activity are frequently observed during filling cystometries. Usually they are attributed either to a neurological disease (1), or to ageing (2). Recently, it has been found that rectal contractions occur more frequently in males with benign prostatic hyperplasia and detrusor overactivity (3). Spontaneous motor activity of the rectum (isolated contraction or sequences of contractions) has been observed and well described during prolonged manometric recordings in both symptomatic and asymptomatic patients $(4,5)$.

The aim of this retrospective study was to review consecutive multichannel urodynamic recordings of women with demonstrated rhythmic rectal contractions (RRCs) in order to determine if there is an association with a specific lower urinary tract symptom (LUTS) and/or a urodynamic finding. 


\section{MATERIALS AND METHODS}

The population consisted of 534 consecutive women with LUTS referred for urodynamics in our laboratory between January 2005 and December 2006. Investigated LUTS were storage (stress, urge and mixed incontinence; increased daytime frequency and nocturia) and voiding symptoms.

The population was divided in two subgroups: 382 patients without a history of neurological disease (non-ND) and 152 with a history of neurological disease (ND), such as stroke, multiple sclerosis, lumbar disc injury, etc. Exclusion criteria were complete spinal cord injury (because the specific physiopathology), inflammatory bowel disease, previous colorectal surgery, rectocele and isolated lower urinary tract pain.

Digital rectal examination allowed to verify that the rectal ampulla was empty. Urodynamics were performed using the Bonito ${ }^{\circledR}$ unit (Laborie Medical Technologies, Paris, France) Filling cystometry was performed at the medium filling rate of $50 \mathrm{~mL} / \mathrm{min}$ (normal saline at room temperature) until maximum bladder capacity in a sitting position. Vesical and urethral pressures were recorded using a triple lumen catheter 10F. Rectal pressure was measured using a punctured balloon filled with $2 \mathrm{~mL}$ of saline solution at room temperature in order to avoid pressure artifacts; the balloon was inserted at $5 \mathrm{~cm}$ of the anal verge. Detrusor pressure was the difference between vesical minus rectal pressure. Pressure transducers were zeroed according with the ICS recommendations (6). Rectal catheter was first inserted and after 2-3 min, then the urethral catheter was inserted. Recordings began about 5 minutes after the insertion. Transmission of pressures was verified by asking the patient to cough before and after the beginning of filling.

Significant RRCs were defined as rhythmic changes in the rectal pressure of at least $3 \mathrm{~cm} \mathrm{H} 2 \mathrm{O}$ independent of the total vesical pressure and independent of respiratory movements.

Occurrence of RRCs was analyzed according to age, neurological history, duration during filling, main complaint and urodynamic diagnosis. Special attention was given to symptoms of urgency (with or without incontinence - overactive bladder - OAB) evaluated from bladder diary and general interroga- tion, and to detrusor overactivity (uninhibited detrusor contractions - DO) during cystometry (7).

Each study was reviewed independently by two investigators (F.V. and B.M.); to be retained, the study needed to be considered univocally positive by both researchers.

For statistical analysis we used Student's-ttest and chi-square test with $\mathrm{p}<0.05$ considered to indicate significance.

\section{RESULTS}

Among the 534 patients (mean age 57.5 years, range 20 to 92 years), 69 (12.9\%) had a rhythmic rectal activity.

Of the 382 patients with no history of neurological disease, 47 (12.3\%) exhibited RRCs compared to the 22 of the 152 patients $(14.4 \%)$ with a history of neurological disease. The neurological disease was suprapontine lesion in 5 patients, incomplete spinal cord lesion in 10 and demyelinating neuropathy in 7. Occurrence of RRCs was not significantly different in the 2 sub-groups: non-ND and ND.

Age was not significantly different between the two sub-groups of women with RRCs; the mean age was $65.5 \pm 15.2$ years in the non-ND group compared to $62.7 \pm 18.0$ years in the ND group. However, women with RRCs were significantly older than the negative population $(64.6 \pm 16.1$ years vs. $56.3 \pm 17.9$ years; $p=0.0002$ ). Figure-1 shows the progressive increase in RRCs incidence with age in our population while the neurological disease incidence is stable or slightly reduced.

RRCs had a low very stable frequency in the range 1 - 4/min; their amplitude was $\leq 15 \mathrm{~cm} \mathrm{H2O}$ in 45 non-ND $(8.2 \pm 4.3 \mathrm{~cm} \mathrm{H} 2 \mathrm{O})$ and $22 \mathrm{ND}$ patients $(8.0 \pm 3.3 \mathrm{~cm} \mathrm{H} 2 \mathrm{O})$, and $>15 \mathrm{~cm} \mathrm{H} 2 \mathrm{O}$ in only 2 nonND patients.

RRCs occurred during all the filling phase in 30 non-ND and 19 ND patients, appeared at first desire to void in only 9 non-ND and disappeared during filling in 8 non-ND and 3 ND patients. Figures 2-4 show examples of the three situations.

RRCs of same characteristics (frequency and amplitude) were observed in 30 (64\%) non-ND and 13 $(59 \%)$ ND patients during pressure recordings before 

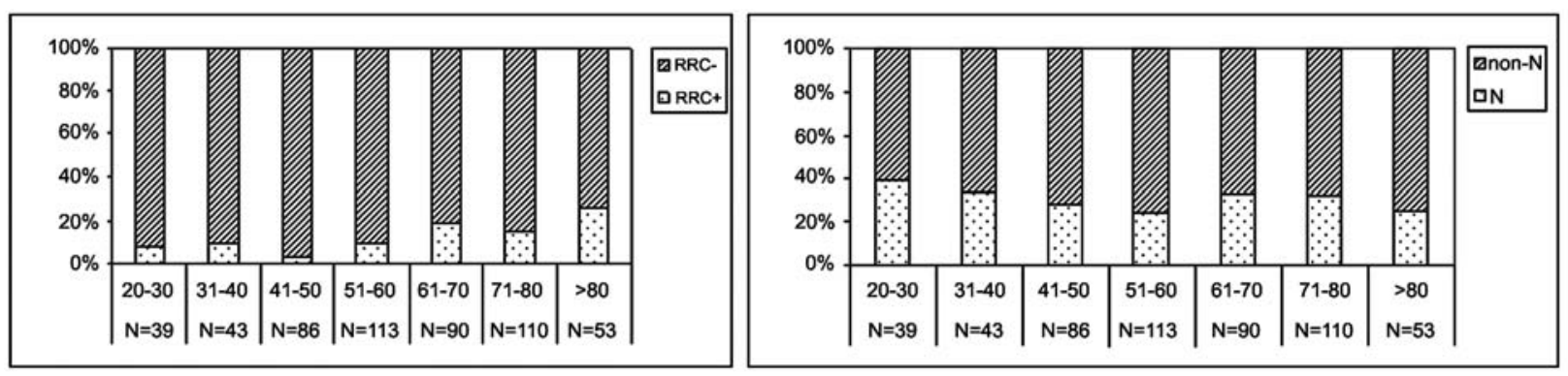

Figure 1 - Analysis of the entire population (534 female patients). Left: incidence of rhythmic rectal contractions with age. Right: incidence of neurological disease with age.

the beginning of filling. That event was independent of the onset, the duration and the end of RRCs during cystometry.

Table- 1 describes the incidence of overactive bladder syndrome and detrusor overactivity.
Comparison with the negative population allowed to find a correlation of RRCs with $\mathrm{OAB}$ $(p=0.0026)$ but not with DO $(p=0.35)$.

Isolated stress urinary incontinence was observed in only $7(14.9 \%)$ non-ND patients (mean

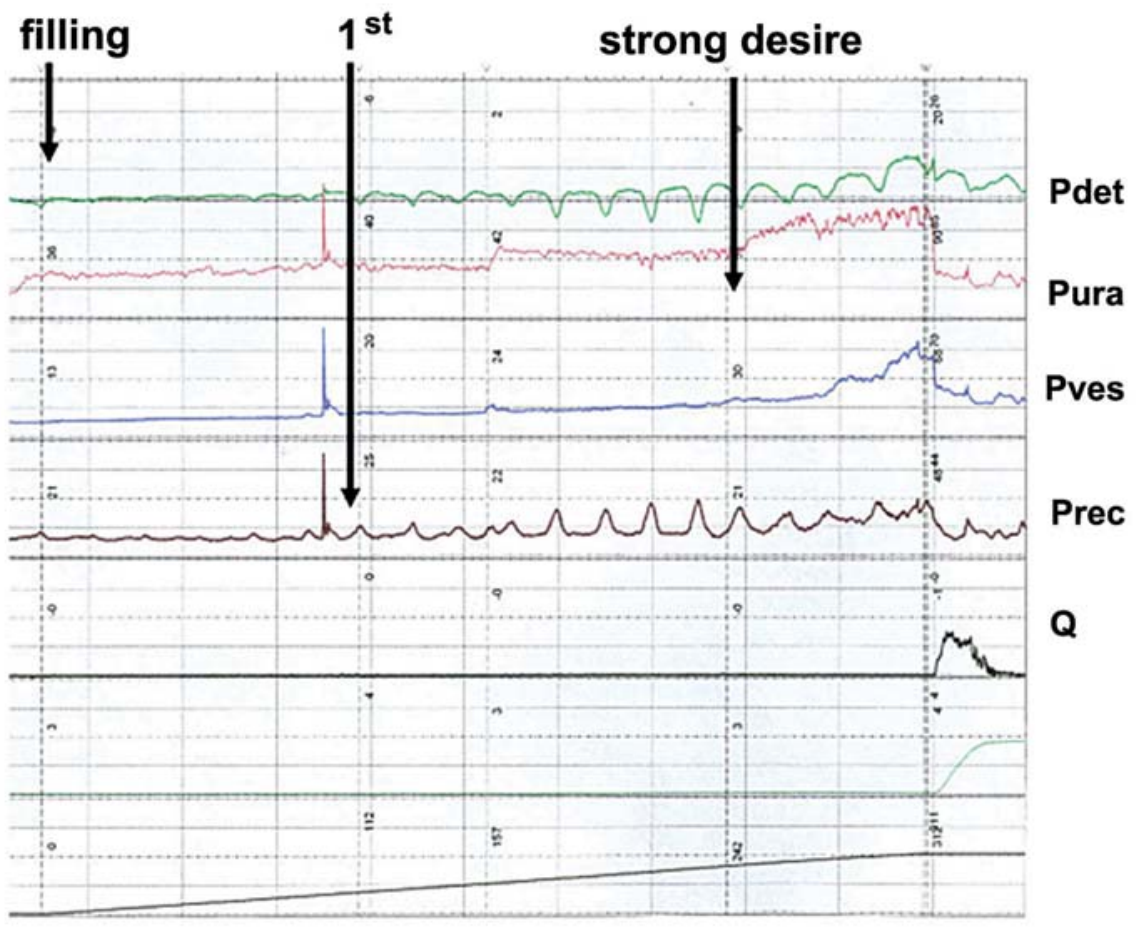

Figure 2 - Example of a patient who had rhythmic rectal contractions after the first desire to void during filling cystometry. 


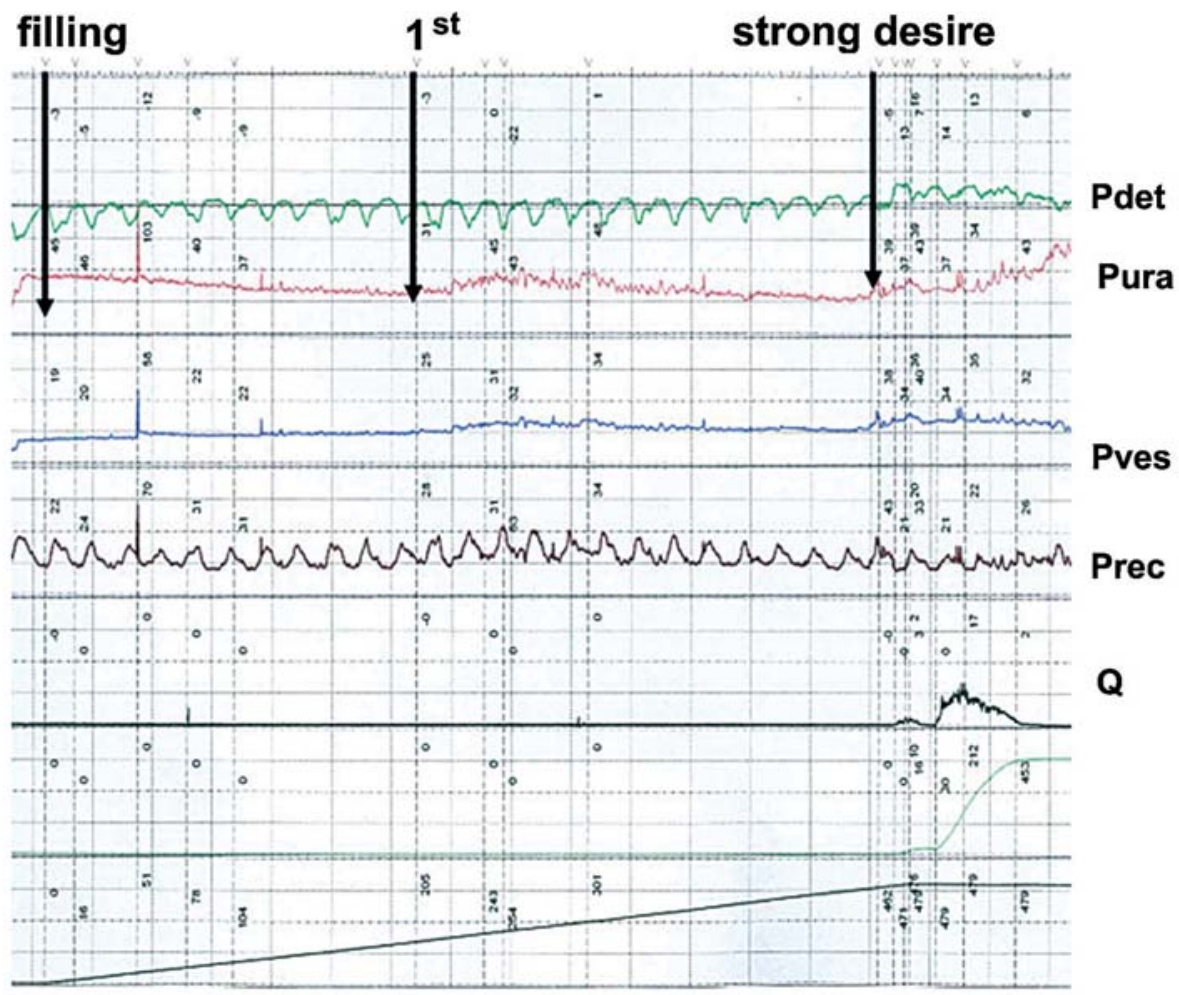

Figure 3 - Example of a patient who had rhythmic rectal contractions during all the filling cystometry.

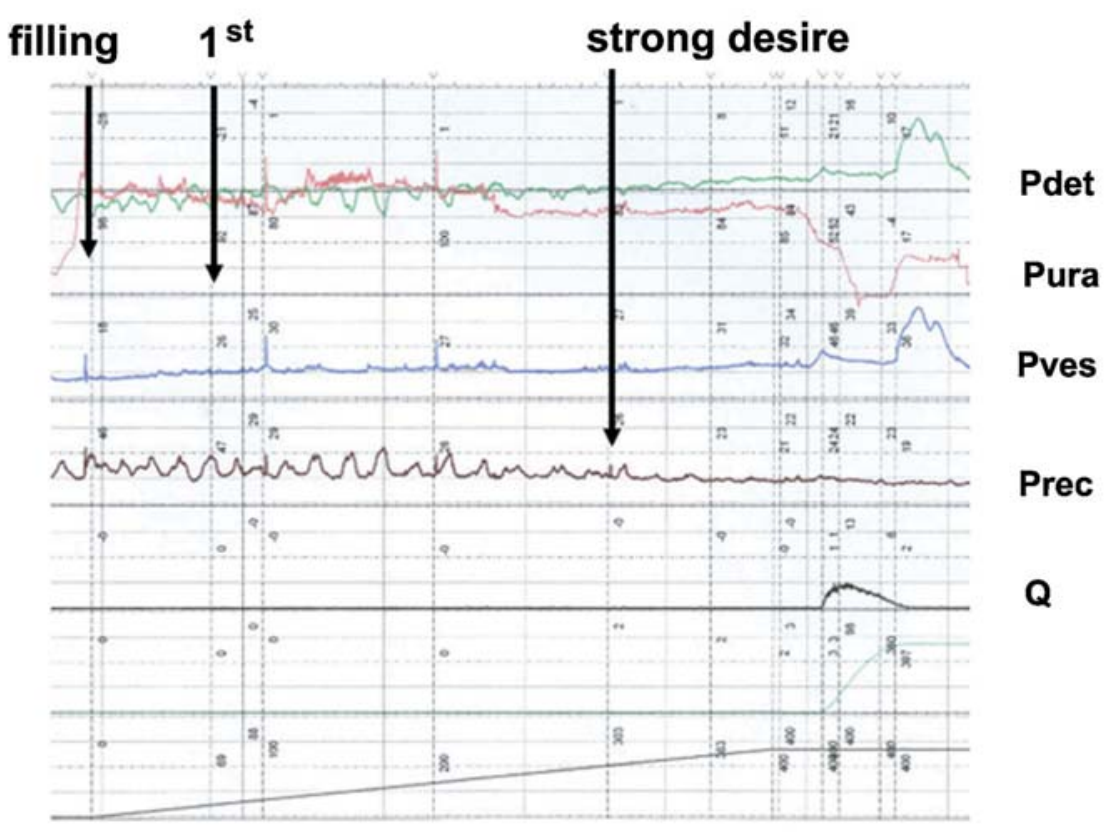

Figure 4 - Example of a patient who had rhythmic rectal contractions until strong desire to void during filling cystometry. 
Table 1 - Incidence of urge syndrome (overactive bladder - OAB) and demonstrated urodynamic detrusor overactivity $(D O)$ in the population with rhythmic rectal contractions.

\begin{tabular}{lccc}
\hline & Non-ND $(\mathrm{N}=37)$ & ND $(22 \mathrm{pts})$ & p Value \\
\hline Urge syndrome $(\mathrm{OAB})$ & $32(68.0 \%)$ & $13(59.1 \%)$ & n.s. \\
DO & $10(21.3 \%)$ & $10(45.5 \%)$ & 0.04 \\
\hline
\end{tabular}

$N D=$ history of neurological disease

age 56.8 years) of whom 5 had RRCs during all the filling phase.

\section{COMMENTS}

Despite the large use of rectal pressure recordings and of Enhörning hypothesis to evaluate the abdominal pressure during urodynamics (detrusor pressure equals vesical pressure minus rectal pressure) only few urodynamicists have paid attention to or studied the rectal activity during cystometries (1-3, $8)$. In all these analyses of rectal contractions during multichannel urodynamic testing, both rhythmic and random rectal contractions have been considered. Our study has two distinctive characteristics. First, we only analyzed the rhythmic rectal contractions and not the random ones; Prior et al. (9) reported that single rectal contractions and bursts of phasic motor activity in the human rectum may have a different origin; filling cystometry is of short duration, less than 20 minutes, which does not allow for an accurate study of random contraction. Second, the population was only an adult female population.

RRCs had a low very stable frequency as if produced by a pacemaker, which led us to compare it with the periodic activity of the gastrointestinal tract. Rhythmic rectal activity has been previously described: runs of powerful phasic contractions (2$3 / \mathrm{min}$ ) or clusters of contractions of relatively low amplitude $(5-6 / \mathrm{min})$ in 12 healthy male volunteers (4) and (3-6/min) in 9 among 11 healthy women (age range 20-46 years) (10). In this study on women, rectal pressure was recorded using a perfused urethral catheter and waves were of higher amplitude (mean
$19 \mathrm{~cm} \mathrm{H} 2 \mathrm{O}$; range 8-54 $\mathrm{cm} \mathrm{H} 2 \mathrm{O}$ ) than in our study. Data regarding the causes and the circadian rhythm of this periodic motor activity remain either lacking or conflicting $(11,12)$. We have observed a rhythmic rectal activity independent of bladder filling in a large proportion of the population with RRCs despite the use to measure the rectal pressure of a punctured balloon, which prevents pressure artifacts arising from contact of the catheter with the rectum wall. That rectal activity is independent of the neurological status. Its characteristics (frequency and amplitude) evoke a phase-III like activity; in the small intestine, the phase III activity (12) is influenced by meal. The periodic rectal motor activity is supposedly triggered by events in the neighboring structures (13). Pezzone et al. (14) have described acute cross-organ sensitization for bladder to bowel and bowel to bladder. That phenomenon may account for chronic pelvic pain disorders. In addition, authors of the same team research (15) have demonstrated the convergence of bladder and colon sensory innervations at the primary afferent level. Therefore, one can consider the role of bladder filling to stimulate the rectum and to evoke a contraction. In fact, disappearance of RRCs during filling was observed in $11(16 \%)$ patients and appearance after first desire in $9(13 \%)$.

In our population, occurrence of RRCs was not significantly associated with a history of neurological disease (14.4\% vs. $12.3 \%)$. That result differs significantly from the findings of Combs and Nitti (1) and Miranne et al. (8) who, studying a population including patients with spinal cord injury and analyzing both rhythmic and random rectal contractions, found respectively when comparing patients with a history of neurological disease vs. patients 
without $51 \%$ vs. $29 \%$ (1) and $32 \%$ vs. $19 \%$ (8). In our sub-group with neurological disease, complaint and urodynamic diagnosis were not homogeneous. Note that the physiopathology of voiding disturbances due to a neurological condition is only well characterized in cases of complete spinal cord lesion.

Occurrence of RRCs is clearly correlated with age as reported by Ozawa et al. despite the small studied group and probably their older population (2). It is known that in elderly, DO might be the consequence of changes in muscarinic receptor subtypes and the non-neural acetylcholine release from urothelium (16). A similar condition with overproduction of acetylcholine from the rectum mucosa could be proposed in elderly, leading to rectal contractions (17). A significant difference is that DO is rarely rhythmic while the studied phenomenon is rhythmic.

We found some other significant results. Urgency is frequently associated with RRCs whatever the neurological status and is more frequent than in the negative population. At the opposite obvious detrusor overactivity during the cystometry is more frequently associated with RRCs in patients with a history of neurological disease. Concerning that last result we have to take into account that several central nervous system disorders are associated with overactive bladder syndrome $(18,19)$ and detrusor overactivity (20).

\section{CONCLUSION}

RRCs cannot be considered as artefactual events during filling cystometry in women with LUTS. RRCs have to be identified during urodynamics due to their involvement in the detrusor pressure calculation. The rhythmic character of the contractions is consistent with the existence of an oscillator. RRCs occur in the older population, are frequently associated with urgency (OAB syndrome) whatever the population (whether or not there is a history of neurological disease). In the population with a history of neurological disease detrusor overactivity is frequent. Occurrence of RRCs must lead the physician to look at possible causes of urgency and detrusor overactivity. Finally, these findings bring to the fore the role of ageing and possibly of the common neurologic innervation of lower urinary and gastrointestinal tracts in the occurrence of rhythmic rectal contractions.

\section{CONFLICT OF INTEREST}

None declared.

\section{REFERENCES}

1. Combs AJ, Nitti VW: Significance of rectal contractions noted on multichannel urodynamics. Neurourol Urodyn. 1995; 14: 73-80.

2. Ozawa H, Nagai A, Akiyama H, Ichikawa T, Akiyama M, Ono N, Oeda T, et al.: Significance of rectal contractions during multichannel urodynamic study. Nippon Hinyokika Gakkai Zasshi. 1997; 88: 874-9.

3. Ghoniem GM, Khater U, Elsergany R, Sakr M. The significance of rectal contractions in benign prostatic obstruction. Urodinamica. 2005; 15: 33-8.

4. Kumar D, Williams NS, Waldron D, Wingate DL: Prolonged manometric recording of anorectal motor activity in ambulant human subjects: evidence of periodic activity. Gut. 1989; 30: 1007-11.

5. Orkin BA, Hanson RB, Kelly KA: The rectal motor complex. J Gastrointest Motil. 1989; 1: 5-8.

6. Djurhuus JC, Navarrete RV, Whitaker R, Griffiths D: Standardization of terminology of the hydrodynamics of the upper urinary tract. International Society for the Dynamics of the Upper Urinary Tract, Subcommittee on Standardization of Terminology. Neurourol Urodyn. 1994; 13: 64754.

7. Abrams P, Cardozo L, Fall M, Griffiths D, Rosier $\mathrm{P}$, Ulmsten $\mathrm{U}$, et al.: The standardisation of terminology of lower urinary tract function: report from the Standardisation Sub-committee of the International Continence Society. Neurourol Urodyn. 2002; 21: 167-78.

8. Miranne JM, Yavagal S, Hernandez-Silen A, Feustel PJ, De EJB. Higher index of suspicion for neurological disease is warranted if rectal con- 
tractions occur during multichannel urodynamic testing. J Urol. 2008; 179(suppl 4): 518. Abstract 1520.

9. Prior A, Fearn UJ, Read NW: Intermittent rectal motor activity: a rectal motor complex? Gut. 1991; 32: 1360-3.

10. Sørensen SM, Gregersen H, Sørensen S, Djurhuus JC: Spontaneous anorectal pressure activity. Evidence of internal anal sphincter contractions in response to rectal pressure waves. Scand J Gastroenterol. 1989; 24: 115-200.

11. Auwerda JJ, Bac DJ, Schouten WR: Circadian rhythm of rectal motor complexes. Dis Colon Rectum. 2001; 44: 1328-32.

12. Rao SS, Welcher K: Periodic rectal motor activity: the intrinsic colonic gatekeeper? Am J Gastroenterol. 1996; 91: 890-7.

13. Kellow JE, Borody TJ, Phillips SF, Tucker RL, Haddad AC: Human interdigestive motility: variations in patterns from esophagus to colon. Gastroenterology. 1986; 91: 386-95.

14. Pezzone MA, Liang R, Fraser MO: A model of neural cross-talk and irritation in the pelvis: implications for the overlap of chronic pelvic pain disorders. Gastroenterology. 2005; 128: 1953-64.
15. Christianson JA, Liang R, Ustinova EE, Davis BM, Fraser MO, Pezzone MA: Convergence of bladder and colon sensory innervation occurs at the primary afferent level. Pain. 2007; 128: 235-43.

16. Andersson KE, Yoshida M: Antimuscarinics and the overactive detrusor--which is the main mechanism of action? Eur Urol. 2003; 43: 15.

17. Sarna SK: Physiology and pathophysiology of colonic motor activity (2). Dig Dis Sci. 1991; 36: 998-1018.

18. Andersson KE, Pehrson R: CNS involvement in overactive bladder: pathophysiology and opportunities for pharmacological intervention. Drugs. 2003; 63: 2595-611.

19. Brading AF: A myogenic basis for the overactive bladder. Urology. 1997; 50(6A Suppl): 57-67; discussion 68-73.

20. Andersson KE: Mechanisms of Disease: central nervous system involvement in overactive bladder syndrome. Nat Clin Pract Urol. 2004; 1: 103-8.

\section{Correspondence address:}

Dr. Françoise A. Valentini

Hôpital Charles Foix

7, avenue de la République

Ivry-sur-Seine, 94200, France

Fax: + 331 4959-4697

E-mail: francoise.valentini@jrs.aphp.fr

\section{EDITORIAL COMMENT}

This is an excellent paper that clearly establish that rhythmic rectal contractions (RRCs) and is of clinical interest and value. For those of us who do urodynamics for a living we all suspect that there is science in the RRC's we have seen but were always sort of shy to speak up because other experts may not believe us. However, since my collaboration with Dr. Mike Pezzone in the field of gastroenterology over the past decade it is becoming obvious to me of the interconnection between urinary and colorectal system (1). 
The take home message is that cross-talk exists between the bladder/urethra and rectum/anus and that RRCs may be a valuable parameter to help us to help our patients with pelvic diseases.

\section{REFERENCE}

1. Pezzone MA, Liang R, Fraser MO: A model of neural cross-talk and irritation in the pelvis: implications for the overlap of chronic pelvic pain disorders. Gastroenterology. 2005; 128: 1953-64.

\author{
Dr. Michael B. Chancellor \\ Neurourology Program \\ Department of Urology \\ William Beaumont Hospital \\ Royal Oak, Michigan, USA \\ E-mail: chancellormb@gmail.com
}

\section{EDITORIAL COMMENT}

This interesting study reported the rhythmic rectal contractions (RRCs) during a urodynamic study in women with lower urinary tract symptoms (LUTS) with or without any neurological lesion. The incidence of RRCs was 69/534 (13\%) without significant difference between those with (14.5\%) and without $(12.3 \%)$ neurological lesion. However, the mean age of women with RRCs was significantly older than those without RRCs. RRCs were also noted to associate with urgency symptoms, but RRCs were associated with occurrence of detrusor overactivity (DO) only in patients with neurological lesions.

In fact, RRCs are not uncommon finding during urodynamic study especially in patients with neurogenic voiding dysfunction. Convergence of the bladder and distal colon sensory innervation had been shown in rat model at dorsal root ganglion neurons. This pelvic organ cross-sensitization may play a role in the overlap of pelvic pain disorders (1). Because rectum and bladder have common neurological reflex arc in sacral cords, therefore, RRCs can be regarded as reflex contractions associated with sensory input through inserting rectal catheter or urethral catheter. If the rectal catheter or urethral catheter is placed for a longer period, RRCs could disappear. The authors might observe the disappearance of RRCs after resting for a longer period. If this phenomenon is observed after a longer period of rest, we should wait for the disappearance of RRCs before starting the urodynamic study to ensure a correct subtracted detrusor pressure.

The results of this study also found a higher incidence of RRCs in older population although there was no significant difference between women with and without neurological lesions. Studies have revealed that the urothelium is involved in sensory mechanisms It has been demonstrated that in the human bladder mucosa, acetylcholine and adenosine triphosphate production from urothelium increased in older patients (2). Intravesical instillation of antimuscarinic agent in rat bladder can block muscarinic receptors in bladder-afferent pathways (3). Older women might also have overproduction of acetylcholine in the mucosa of rectal wall, which might also result in spontaneous contractions through stretching of the rectal wall after inserting rectal catheter. Moreover, chronic constipation is a more frequent disorder in the elderly. It is possible that the elderly women have a higher incidence of chronic constipation, which in turn causes frequent RRCs in response to rectal stimulation by the rectal catheter.

The bladder and rectum are located at the convergence area in the sacral cord. The sensation of one organ (bladder) can be induced by stimulating the 
other organ (rectum). Therefore, rectal stimulation might evoke sensory response (urgency sensation) or DO in the urinary bladder and hence resulting in an urgency sensation during urodynamic study. Therefore, it is not surprising that occurrence of RRCs is associated with urgency sensation in overall patients and with DO in patients with neurological lesion.

The clinical implication of RRCs might be the provocation of sensory urgency or DO in women who have a hyperactive rectum during natural bladder filling. This phenomenon may be observed in some patients with chronic constipation and overactive bladder symptoms due to this hyperactive rectum and RRCs in their daily life. Based on the findings of this study and the possible mechanism of RRCs, we should not consider RRCs as artefactual events during cystometry. Moreover, in cases of RRCs occurrence without concomitant urgency or DO (as in 34 patients in this study) during the urodynamic study, the disordered rectum might be considered as the origin of their LUTS. Treatment with antimuscarinic might also be beneficial in these patients.

\section{REFERENCES}

1. Christianson JA, Liang R, Ustinova EE, Davis BM, Fraser MO, Pezzone MA: Convergence of bladder and colon sensory innervation occurs at the primary afferent level. Pain. 2007; 128: 235-43.

2. Yoshida M, Miyamae K, Iwashida H, Otani M, Inadome A: Management of detrusor dysfunction in the elderly: Changes in acetylcholine and adenosine triphosphate release during aging. Urology. 2004; 63 (3 Suppl 1): 17-23.

3. Kim Y, Yoshimura N, Masuda H, de Miguel F, Chancellor MB: Antimuscarinic agents exhibit local inhibitory effects on muscarinic receptors in bladder-afferent pathways. Urology. 2005; 65: 238-42.

Dr. Hann-Chorng Kuo Department of Urology

Buddhist Tzu Chi General Hospital and Tzu Chi University Hualien, Taiwan E-mail:hck@tzuchi.com.tw 